\title{
EXAFS study of zinc coordination in bacitracin A
}

\author{
Finn Drabløs ${ }^{\mathrm{a}, *}$, David G. Nicholson ${ }^{\mathrm{b}}$, Magnus Rønning ${ }^{\mathrm{c}}$ \\ a SINTEF Unimed MR Centre, N-7465 Trondheim, Norway \\ b Department of Chemistry, Norwegian University of Science and Technology (NTNU), N-7491 Trondheim, Norway \\ c Department of Industrial Chemistry, Norwegian University of Science and Technology (NTNU), N-7491 Trondheim, Norway
}

Received 27 November 1998; received in revised form 18 February 1999; accepted 9 March 1999

\begin{abstract}
Bacitracin is a dodecapeptide antibiotic produced by Bacillus sp. The antibacterial activity depends upon the peptide binding a divalent metal. Hitherto, the exact coordination of the cation has not been established. In particular the role played by the sulphur and nitrogen atoms of the thiazoline ring of bacitracin $\mathrm{A}$ has not been clear. Here the coordination of $\mathrm{Zn}^{2+}$ by bacitracin A has been studied using extended X-ray absorption fine structure. The experimental data are consistent with a model in which zinc is coordinated by one oxygen and three nitrogen atoms with the sulphur atom of the thiazoline ring not being directly involved in the zinc coordination. (C) 1999 Elsevier Science B.V. All rights reserved.
\end{abstract}

Keywords: Bacitracin A; Thiazoline; Zinc; Extended X-ray absorption fine structure

\section{Introduction}

Bacitracins are macrocyclic dodecapeptides produced by Bacillus subtilis and Bacillus licheniformis [1]. They show antibiotic activity against many Gram-positive and a few Gram-negative species of bacteria, and although the detailed nature of this activity has not been clarified, it is believed that bacitracin inhibits cell wall synthesis by complexation of the pyrophosphate form of a $\mathrm{C}_{55}$-isoprenyl carrier lipid $[2,3]$. The main form of the peptide is bacitracin A (Fig. 1) [4].

\footnotetext{
Abbreviations: CSD, Cambridge Structural Database; EPR, electron paramagnetic resonance; EXAFS, extended X-ray absorption fine structure; FT, Fourier transform; NMR, nuclear magnetic resonance; SERC, Science and Engineering Research Council; XAS, X-ray absorption spectroscopy

* Corresponding author. Fax: +47 (73) 867708;

E-mail: finn.drablos@unimed.sintef.no
}

Although bacitracins are peptides, they are not synthesised by the standard ribosomal pathway. A large synthetase complex builds the peptide by connecting individual fragments. The genome sequence of this synthetase complex has recently been determined [5]. As a consequence of the non-standard pathway, bacitracin A exhibits some unusual features not commonly found in peptides. Four out of 12 residues have $\mathrm{D}$ chirality, rather than the standard $\mathrm{L}$ form. A thiazoline ring is formed by cyclisation of the cysteine side chain, and a macrocyclic ring is formed by coupling the C-terminal end at Asn-12 to the side chain of Lys-6. Bacitracin A also includes an ornithine residue.

It has been shown that a divalent metal ion is important for antibiotic activity of bacitracin A [6], probably because the metal ion is necessary for complex formation between bacitracin A and the carrier lipid pyrophosphate. It is generally assumed that this metal ion is $\mathrm{Zn}^{2+}$, which has been shown to give 


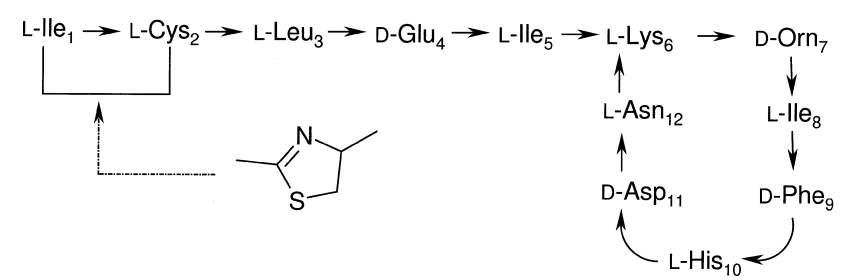

Fig. 1. Primary structure of bacitracin A. The thiazoline ring is formed by cyclisation between Ile-1 and Cys-2, whereas a peptide-type bond between Asn-12 and Lys-6 forms the macrocyclic ring.

optimal binding of carrier lipid pyrophosphate [7]. The structure of bacitracin A has been studied both by X-ray [8,9] and by nuclear magnetic resonance (NMR) [10]. However, these studies do not show how bacitracin binds cations. The crystal structures are for complexes between bacitracin A and proteases, and in one case where crystallisation was done from zinc-bacitracin, the zinc ion was released from bacitracin upon formation of the bacitracinprotease complex. This was confirmed by extended X-ray absorption fine structure (EXAFS) [9], but no detailed information about the zinc coordination in bacitracin A from these data has been published.

Several studies have investigated individual residues in bacitracin A that are involved in binding of cations, and these studies are summarised below.

\subsection{Histidine (His-10)}

Several independent experiments have shown that the imidazole group of histidine is involved in the metal binding [7,11-17].

\subsection{Aspartic acid (Asp-11)}

In some studies it has been suggested that the carboxyl group of aspartic acid is involved in metal ion binding. An early NMR study by Wasylishen and Graham [14] used this interpretation to explain line broadening effects produced by the presence of paramagnetic copper ions, and a subsequent electron paramagnetic resonance (EPR) study by Seebauer et al. [17] on the same system reached a similar conclusion. However, in contrast with these observations a NMR study by Mosberg et al. [15] using $\mathrm{Zn}^{2+}$ as the complexed ion showed no significant effect on the aspartic carboxyl fragment. A possible explanation for this variation may be differences between the coordinating properties of $\mathrm{Cu}^{2+}$ and $\mathrm{Zn}^{2+}$. Based on the data by Mosberg et al. we will assume that the carboxyl group of the aspartic acid does not coordinate to zinc.

\subsection{Glutamic acid (Glu-4)}

Mosberg et al. [15] showed that the addition of zinc ions strongly affects the carboxyl group of glutamic acid. This is also consistent with studies using cations other than $\mathrm{Zn}^{2+}[14,17]$. Thus, from these studies a likely conclusion is that most metal ions bound by bacitracin are coordinated by the carboxyl group of glutamic acid. This may also explain why zinc-bacitracin releases zinc upon formation of a bacitracin-protease complex [9], as the published crystal structures show that Glu-4 is in direct interaction with active site residues of the protease in this complex.

\subsection{Thiazoline (Cys-2)}

Several independent studies show that the thiazoline ring is involved in coordinating cations [12$15,17]$. However, it has not been possible to ascertain whether these cations bind to nitrogen or sulphur of the thiazoline ring. Mosberg et al. reported a large downfield shift in the NMR resonances of the methylene protons close to the sulphur atom of the thiazoline ring [15], and this was used as an argument for zinc being coordinated by the sulphur atom. Seebauer et al. in their EPR study ruled out coordination to sulphur [17]. However, since this experiment was carried out on a $\mathrm{Cu}^{2+}$ system this conclusion is not unequivocal with respect to the $\mathrm{Zn}^{2+}$ system (see above).

\section{5. $\mathrm{N}$-Terminal $-\mathrm{NH}_{2}$ (Ile-1)}

Another group that may be involved in coordination of cations is the $\mathrm{N}$-terminal $-\mathrm{NH}_{2}$ group. Several experiments have shown that this group is strongly affected by metal ions $[7,15,18]$. It has been suggested that the $\mathrm{p} K_{\mathrm{a}}$ of this group is shifted from 7.7 to 5.7 upon metal ion binding [16], and the presence of a group with $\mathrm{p} K_{\mathrm{a}}$ near 5.5 in the metal-bacitracin complex has been confirmed experimentally [18]. How- 
ever, proton release data $[16,18]$ show that approx. 1.5 protons are released when metal ion is added at pH 5.5, and approx. 0.6 protons can be titrated with $\mathrm{NaOH}$ after the addition of metal ion. The interpretation is that only one proton is released as a direct consequence of interaction with metal ion, and that the rest is a consequence of a change in $\mathrm{p} K_{\mathrm{a}}$ of the $\mathrm{N}$-terminal group. However, the binding studies of Storm and Strominger [7] show that the optimal $\mathrm{pH}$ region for binding metal-bacitracin $\mathrm{A}$ to $\mathrm{C}_{55}$-isoprenyl pyrophosphate ranges from $\mathrm{pH} 6$ to 9. They also concluded, using a different argument than that forwarded by Scogin et al., that the N-terminal group could not be involved in metal ion binding. As the $\mathrm{p} K_{\mathrm{a}}$ of a free $\mathrm{N}$-terminal $-\mathrm{NH}_{2}$ is close to 9.0 , it is protonated in the optimal binding region of bacitracin, and therefore cannot interact with the positively charged metal ion. However, if the hypothesis about a $\mathrm{p} K_{\mathrm{a}}$ shift is correct, it will not be protonated in this $\mathrm{pH}$ range, and can very well participate in coordinating the metal ion. One possible explanation is that the interaction between the metal ion and the $\mathrm{N}$-terminal $-\mathrm{NH}_{2}$ is relatively weak, so that it is easily displaced either by titration or by the pyrophosphate group, in which case the protonated form of the $-\mathrm{NH}_{2}$ group may actually participate in binding the pyrophosphate, whereas the unprotonated form will bind the metal ion.

\subsection{Water}

There is no experimental information regarding the possible involvement of water molecules in the coordination sphere of the zinc ion in bacitracin. However, it is a definite possibility that water molecules may be involved, either as an additional ligand or by substituting the $\mathrm{N}$-terminal $-\mathrm{NH}_{2}$ as a zinc ligand.
There are few naturally occurring analogues to bacitracin for which there are experimental data. However, the structure of one relevant molecule has recently been published, namely farnesyl transferase [19]. This enzyme is involved in connecting farnesyl isoprenoid to cellular signal transduction proteins, the binding of farnesyl pyrophosphate being the initial step in this process. The molecule is thus a catalytic analogue to bacitracin. It has a zinc binding site involved in pyrophosphate binding, and the zinc ion is coordinated by the imidazole ring of histidine, the sulphur atom of cysteine, the carboxylic side chain of an aspartic acid, and a water molecule. The question about sulphur (S) vs. nitrogen (N) coordination for the interaction between thiazoline and zinc may thus have implications for understanding the properties of bacitracin compared to other molecules, in particular with respect to any potential catalytic activity of bacitracin.

The data described above give us several alternative models for how bacitracin A may bind a metal ion like $\mathrm{Zn}^{2+}$, as shown in Table 1. Here we have assumed that the zinc ion is at least tetra-coordinated, as zinc structures with less than four ligands are very uncommon according to data found in a screening of the Cambridge Structural Database (CSD) (vide infra).

The alternatives in Table 1 describe the atoms directly involved in coordinating the metal ion. As a consequence of the structure of the groups involved, we know that just outside this first coordination shell there will be an additional oxygen atom from the glutamic acid, a nitrogen atom from the imidazole ring of histidine and a nitrogen or a sulphur atom from the thiazoline ring, as well as carbon atoms.

We report here the results of an EXAFS study on the local environment about the zinc ion in the zincbacitracin A complex.

Table 1

Alternative coordination modes for zinc in bacitracin A

\begin{tabular}{llllll}
\hline Group & Ligand & & & & \\
\hline$-\mathrm{NH}_{2}$ & $\mathrm{~N}$ & & $\mathrm{~N}$ & $\mathrm{~N}$ & $\mathrm{~N}$ \\
Thiazoline & $\mathrm{S}$ & $\mathrm{S}$ & $\mathrm{S}$ & $\mathrm{N}$ & $\mathrm{N}$ \\
Glu & $\mathrm{O}$ & $\mathrm{O}$ & $\mathrm{O}$ & $\mathrm{O}$ & $\mathrm{O}$ \\
$\mathrm{His}$ & $\mathrm{N}$ & $\mathrm{N}$ & $\mathrm{N}$ & $\mathrm{N}$ & $\mathrm{N}$ \\
$\mathrm{H}_{2} \mathrm{O}$ & & $\mathrm{O}$ & $\mathrm{O}$ & $\mathrm{O}$ & $\mathrm{O}$ \\
$\mathrm{Name}$ & $\mathrm{NSON}$ & $\mathrm{SONO}$ & $\mathrm{NSONO}$ & $\mathrm{NNON}$ & $\mathrm{NONO}$ \\
\hline
\end{tabular}




\section{Material and methods}

\subsection{Statistics on bond lengths}

The CSD [20] version 5.15 (April 1998) was screened using Quest3D. Statistics on bond lengths were generated from the Quest3D output using Vista version 2.1.

\subsection{X-Ray absorption data collection}

Bacitracin zinc salt, $\mathrm{C}_{66} \mathrm{H}_{101} \mathrm{~N}_{17} \mathrm{O}_{16} \mathrm{SZn}$ (B. subtilis) from Fluka was used without further purification. Any contamination will mainly be from bacitracin $B_{1}, B_{2}$ and $B_{3}$, which can be assumed to bind zinc in the same way as bacitracin $\mathrm{A}$, and bacitracin $\mathrm{F}_{\mathrm{A}}$, which will not bind zinc strongly [7].

X-Ray absorption spectroscopy (XAS) data were collected in the transmission mode on station 7.1, Daresbury Laboratory SRS, UK with an electron beam energy of $2 \mathrm{GeV}$ and an average beam current of $200 \mathrm{~mA}$. This station is equipped with an ordersorting $\mathrm{Si}$ (III) monochromator that was offset to $50 \%$ of the rocking curve for harmonic rejection. An unfocussed beam of dimensions $0.8 \mathrm{~mm}$ vertically and $10 \mathrm{~mm}$ horizontally was used. Room temperature zinc K-edge data $(E=9661 \mathrm{eV}, \lambda=1.28330 \AA)$ were registered over the energy range $9510-10410 \mathrm{eV}$. Gas ion chambers were used to detect the intensities of the incident and transmitted X-rays. The first ion chamber was filled with $20 \% \operatorname{Ar}$ (56 Torr) and the second ion chamber with $80 \%$ Ar (406 Torr) and both filled to atmospheric pressure with helium.

Energy calibrations were effected by regularly measuring the spectrum of a $\mathrm{Zn}$ foil (thickness 5 $\mu \mathrm{m}$; the energy of the first deflection being defined as $9661 \mathrm{eV})$. The XAS of bacitracin and the model/ reference compounds zinc sulphide (zinc blende), zinc formate dihydrate and zinc tetraphenylporphyrin were measured. The amounts of material in the samples for XAS were calculated from element mass fractions and the absorption coefficients of the constituent elements [21], just above the absorption edge to give an absorber thickness of 1.5 absorption lengths.

Spectra were measured on well-powdered samples intimately mixed with boron nitride to give a sample thickness of approx. $1.0 \mathrm{~mm}$, placed in aluminium sample holders and held in place by Kapton windows. Nine scans of each compound were collected and summed to give the final spectra.

\section{Results and discussion}

\subsection{Statistics on bond lengths}

In order to compare alternative solutions we need data on the most common bond lengths for relevant $\mathrm{Zn}^{2+}$-ligand interactions. The CSD was screened for model compounds containing subgroups directly bonded to $\mathrm{Zn}$, as shown by the search templates in Table 2, and the results are shown in the table. The bonds defined in the CSD entries were accepted without further verification. However, in almost all cases this approach gave the same result as using contacts to zinc ions identified by atom-atom distances less than the sum of the van der Waals radii for the atoms, as defined in Quest3D [22]. Only error-free entries with an $R$-factor $\leq 10 \%$ were used. Results for tetra-, penta- and hexa-coordinated zinc are shown, as an initial screening of the database showed that this accounted for $93 \%$ of all entries in the database. All data are included in the statistics, without pre-screening for potential outliers.

In general, the distances listed for the same ligand involved in complexes with different coordination numbers are not significantly different, although there is a clear trend showing increasing bond length with increasing coordination number. However, when we compare distances for different ligands, in particular sulphur-based ligands vs. nitrogen-based ligands, it is easy to find significant differences.

\subsubsection{The $\mathrm{Zn}-\mathrm{S}$ distance (thiazoline)}

For the interaction between $\mathrm{Zn}$ and $\mathrm{S}$ of a thiazoline ring there is a paucity of data. From a chemical point of view the group used as model system in model 2 seems to be the one most similar to the situation in thiazoline. However, the average bond length is relatively long, and in particular it is longer than the bond length found in the model system used for EXAFS analysis. Model 3 represents an alternative bonding mode; in this model there is double bond character in $\mathrm{S}$. In this case the $\mathrm{Zn}-\mathrm{S}$ bond length is reduced compared to model 2 , and it is 
Table 2

Zinc-ligand distances in zinc coordination for ligands from CSD, identified using the listed search templates

\begin{tabular}{|c|c|c|c|c|c|c|c|}
\hline Model & Template & Ligand & Coord & $\mathrm{n}$ & Median & Average & $\mathrm{SD}$ \\
\hline \multirow{3}{*}{1} & \multirow{3}{*}{$\mathrm{S}-\mathrm{Zn}$} & \multirow{3}{*}{ S } & 4 & 305 & 2.32 & 2.33 & 0.06 \\
\hline & & & 5 & 172 & 2.37 & 2.44 & 0.15 \\
\hline & & & 6 & 43 & 2.55 & 2.54 & 0.10 \\
\hline \multirow{3}{*}{2} & \multirow{3}{*}{$\mathrm{C}_{\mathrm{S}}^{\mathrm{C}_{\mathrm{S}}-\mathrm{Zn}}$} & \multirow{3}{*}{$\mathrm{S}$} & 4 & 2 & & 2.40 & \\
\hline & & & 5 & 6 & 2.66 & 2.66 & 0.05 \\
\hline & & & 6 & 14 & 2.61 & 2.60 & 0.09 \\
\hline \multirow{3}{*}{3} & \multirow{6}{*}{$N-Z n$} & \multirow{3}{*}{$\mathrm{S}$} & 4 & 56 & 2.34 & 2.34 & 0.04 \\
\hline & & & 5 & 19 & 2.37 & 2.41 & 0.10 \\
\hline & & & 6 & 3 & 2.52 & 2.51 & \\
\hline \multirow{3}{*}{4} & & \multirow{3}{*}{$\mathrm{N}$} & 4 & 1022 & 2.04 & 2.05 & 0.06 \\
\hline & & & 5 & 898 & 2.09 & 2.11 & 0.09 \\
\hline & & & 6 & 600 & 2.16 & 2.16 & 0.08 \\
\hline \multirow{3}{*}{5} & \multirow{3}{*}{$\mathrm{N}^{\mathrm{N}}-\mathrm{Zn}$} & \multirow{3}{*}{$\mathrm{N}$} & 4 & 262 & 2.03 & 2.03 & 0.03 \\
\hline & & & 5 & 186 & 2.06 & 2.08 & 0.06 \\
\hline & & & 6 & 50 & 2.09 & 2.11 & 0.08 \\
\hline \multirow{3}{*}{6} & & \multirow{3}{*}{$\mathrm{N}$} & 4 & 8 & 2.04 & 2.03 & 0.03 \\
\hline & & & 5 & 2 & & 2.03 & \\
\hline & & & 6 & 4 & 2.13 & 2.12 & 0.17 \\
\hline \multirow{3}{*}{7} & & \multirow{3}{*}{$\mathrm{N}$} & 4 & 13 & 2.04 & 2.03 & 0.04 \\
\hline & & & 5 & 53 & 2.08 & 2.10 & 0.08 \\
\hline & & & 6 & 54 & 2.18 & 2.18 & 0.09 \\
\hline \multirow{3}{*}{8} & & \multirow{3}{*}{$\mathrm{N}$} & 4 & 56 & 2.02 & 2.02 & 0.03 \\
\hline & & & 5 & 21 & 2.01 & 2.03 & 0.06 \\
\hline & & & 6 & 4 & 2.08 & 2.08 & 0.12 \\
\hline \multirow{3}{*}{9} & \multirow{3}{*}{$\mathrm{O}-\mathrm{Zn}$} & \multirow{3}{*}{$\mathrm{O}$} & 4 & 522 & 1.98 & 1.99 & 0.08 \\
\hline & & & 5 & 356 & 2.03 & 2.07 & 0.13 \\
\hline & & & 6 & 649 & 2.10 & 2.13 & 0.11 \\
\hline \multirow{3}{*}{10} & & \multirow{3}{*}{$\mathrm{O}$} & 4 & 109 & 1.96 & 1.96 & 0.03 \\
\hline & & & 5 & 92 & 2.01 & 2.03 & 0.09 \\
\hline & & & 6 & 103 & 2.09 & 2.11 & 0.08 \\
\hline \multirow{3}{*}{11} & \multirow{3}{*}{$\mathrm{H}_{\mathrm{O}}^{\mathrm{H}}-\mathrm{Zn}$} & \multirow{3}{*}{$\mathrm{O}$} & 4 & 0 & & & \\
\hline & & & 5 & 8 & 2.02 & 2.05 & 0.07 \\
\hline & & & 6 & 64 & 2.09 & 2.10 & 0.06 \\
\hline
\end{tabular}

quite close to the general average $\mathrm{Zn}-\mathrm{S}$ bond length found in model 1 . Ab initio computations on a model system (2,4-dimethyl-4,5-dihydrothiazole in complex with $\left.\mathrm{Zn}^{2+} \cdot\left(\mathrm{NH}_{3}\right)_{3}\right)$ at the B3LYP/6-31G** level show that the $\mathrm{Zn}-\mathrm{S}$ distance may be slightly reduced in potential zinc-thiazoline interactions, compared to structures similar to model 2, and that it probably is closer to $2.36 \AA$ (F. Drabløs, unpublished data). This indicates that the bond length in model 2 may be slightly too long to be used as a model system for 
this interaction. However, model 3 would mean ring opening of the thiazoline ring, which does not seem to be the case. The most obvious conclusion is therefore that we do not have a proper model system for the $\mathrm{Zn}-\mathrm{S}$ bond length in the zinc-thiazoline interaction, although the nature of the thiazoline ring seems to indicate that model 3 defines the lower limit of the bond length range. It therefore seems unlikely that the $\mathrm{Zn}-\mathrm{S}$ bond length is shorter than approx. $2.3 \AA$, which is also shorter than the average bond length for tetra-coordinated compounds found for model 1. Based on the data for models 1 and 3, a bond length of $2.5 \AA$ may be a reasonable upper limit for an expected bond length, in particular if the coordination number turns out to be lower than 6 .

There is an obvious lack of structural data on interactions between thiazoline ring sulphur atoms and any metal ion. Screening CSD for all entries with a transition metal coordinated by a thiazoline ring returned 19 entries where the metal ion was bound to the thiazoline nitrogen, but no entries where the metal ion was bound to the corresponding sulphur atom. Thus, it would appear that the nitrogen atom of the thiazoline ring is the preferred coordination site for metal ions.

There are several interesting examples of this preference. Shuter et al. [23] have studied complexes between 2-(2'-hydroxyphenyl)-2-thiazoline and technetium. Rotation around the phenyl-thiazoline bond can bring both $\mathrm{S}$ and $\mathrm{N}$ of thiazoline into identical orientations with respect to the hydroxy group. Yet the crystal structure shows only binding to the nitrogen atom in the thiazoline ring. The same can be observed for thiazole complexes, as shown e.g. by Boo et al. [24] and by Khalil et al. [25].

The reason for this preference may be attributed to the delocalisation of electrons in this type of ring systems, as shown in Fig. 2. This type of delocalisation is observed in oxazolines [26], and in thiazoles [27] and although it is normally not seen in free thiazolines, the changes in bond lengths observed upon complexation with a metal ion are consistent with this type of delocalisation (see [23]).

\subsubsection{The $\mathrm{Zn}-\mathrm{N}$ distance (thiazoline)}

For coordination of $\mathrm{Zn}$ to $\mathrm{N}$ in the thiazoline ring there are two relevant model systems (models 5 and 6 ). The most reliable model is probably model 5 with

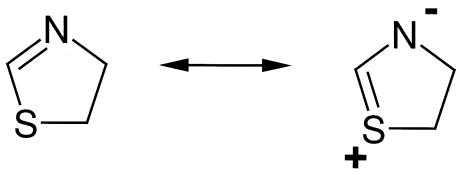

Fig. 2. Resonance structures of thiazoline. A positive charge interacting with the nitrogen will stabilise the charge separation.

a bond length of $(2.03 \pm 0.03) \AA$ at 1 S.D. for a tetracoordinated system. Model 6 was tested in order to allow for the possible interaction between the double bond and the lone pairs of $\mathrm{S}$ in the thiazoline ring, which will change the properties of the ligand. Due to the small number of related compounds both $\mathrm{S}$ and $\mathrm{O}$ were used when searching for model structures. There is no significant reduction in the $\mathrm{Zn}-\mathrm{N}$ bond length, and model 5 is therefore used.

\subsubsection{The $\mathrm{Zn}-\mathrm{N}$ distance ( $\mathrm{N}$-terminal $-\mathrm{NH}_{2}$ )}

For the coordination to the $\mathrm{N}$-terminal $-\mathrm{NH}_{2}$ group we have a good data set in model 7 , and an average bond length of $(2.03 \pm 0.03) \AA$ for a tetracoordinated system seems reasonable.

\subsubsection{The $Z n-N$ distance (histidine)}

Coordination to an imidazole ring or a similar system is also well represented (model 8 ). This gives an average bond length of $(2.02 \pm 0.03) \AA$.

\subsubsection{The Zn-O distance (glutamic acid)}

Coordination of $\mathrm{Zn}$ by a $\mathrm{C}_{-} \mathrm{COO}^{-}$group is well represented by the data set in model 10 , which gives an average length of $(1.96 \pm 0.03) \AA$. This bond length may be slightly biased by cases were both oxygens are used for coordination, but at different distances. However, this effect seems to be small, and we have not tried to correct for it.

\subsubsection{The $\mathrm{Zn}-\mathrm{O}$ distance (water)}

Water molecules seem to be very uncommon in the coordination sphere of tetra- and penta-coordinated zinc ions. The distances found are very similar to general $\mathrm{Zn}-\mathrm{O}$ distances.

The bond lengths identified here (for tetra-coordinated zinc complexes $\mathrm{Zn}-\mathrm{S} \quad 2.3-2.5 \AA$; $\mathrm{Zn}-\mathrm{N}$ $2.03 \pm 0.03, \quad 2.03 \pm 0.04, \quad 2.02 \pm 0.03 ; \quad \mathrm{Zn}-\mathrm{O} \quad 1.96 \pm$ $0.03)$ are comparable to distances found in the reference compounds used in the EXAFS data analysis (Zn-S 2.35 Aं; Zn-N 2.037; Zn-O 2.107) (vide infra). 
The distances are also comparable to ab initio distances in the related model system described above (Zn-S 2.36 ̊; Zn-N 1.98; Zn-NH 3 2.07) (F. Drabløs, unpublished data).

\subsection{EXAFS data analysis}

The data were corrected for dark currents, converted to $k$-space, summed and background subtracted to yield the EXAFS function $\chi_{i}^{\text {obs }}(k)$ using the EXCALIB and EXBACK programmes [28]. Model fitting was carried out with EXCURV90 using curved-wave theory and ab initio phase shift [29], with the edge positions being determined from the first inflection points of the derivative spectra. The phase shifts experienced by the photoelectron, the amplitude of the backscattering and the electron mean free path were calculated from within EXCURV90 using spherical wave theory and a single scattering model.

The model compounds zinc sulphide $\left(\mathrm{Zn}-\mathrm{S}_{4} 2.35\right.$ A) [30], zinc formate dihydrate $\left(\mathrm{Zn}-\mathrm{O}_{6} 2.107 \AA\right.$, averaged) [31] and zinc tetraphenylporphyrin $\left(\mathrm{Zn}-\mathrm{N}_{4}\right.$ $2.037 \AA$, averaged) [32] were used to check the validity of the ab initio phase shifts and amplitudes for sulphur, oxygen and nitrogen backscatterers respectively, and to establish the general parameters, AFAC (proportion of absorption causing EXAFS) and VPI (allows for inelastic scattering of the photoelectron) [29]. During least-squares fitting it is important to avoid correlation effects between those parameters which strongly affect the EXAFS amplitude and between those that influence the frequency of the EXAFS oscillations. Therefore, the EXAFS spectra were least-squares fitted using $k^{1}$ and $k^{3}$ weighted data because, as has been shown [33], optimising the $k^{1}$ and $k^{3}$ weighted fits reduces the degree of coupling between the two highly correlated sets of parameters $\left(N\right.$, AFAC, $2 \sigma^{2}$ and $\left.R, E_{0}\right)$ giving a solution common to both weighting schemes, where $N$ is the multiplicity, AFAC the amplitude reduction factor, $2 \sigma^{2}$ the Debye-Waller-type factor, $R$ the distance and $E_{0}$ the magnitude of the photoelectron energy at zero wave vector.

The $k^{3}$ weighting scheme used in the refinement compensates for the diminishing photoelectron wave at higher $k$. Curve fitting was performed on data that had been Fourier filtered over a wide range

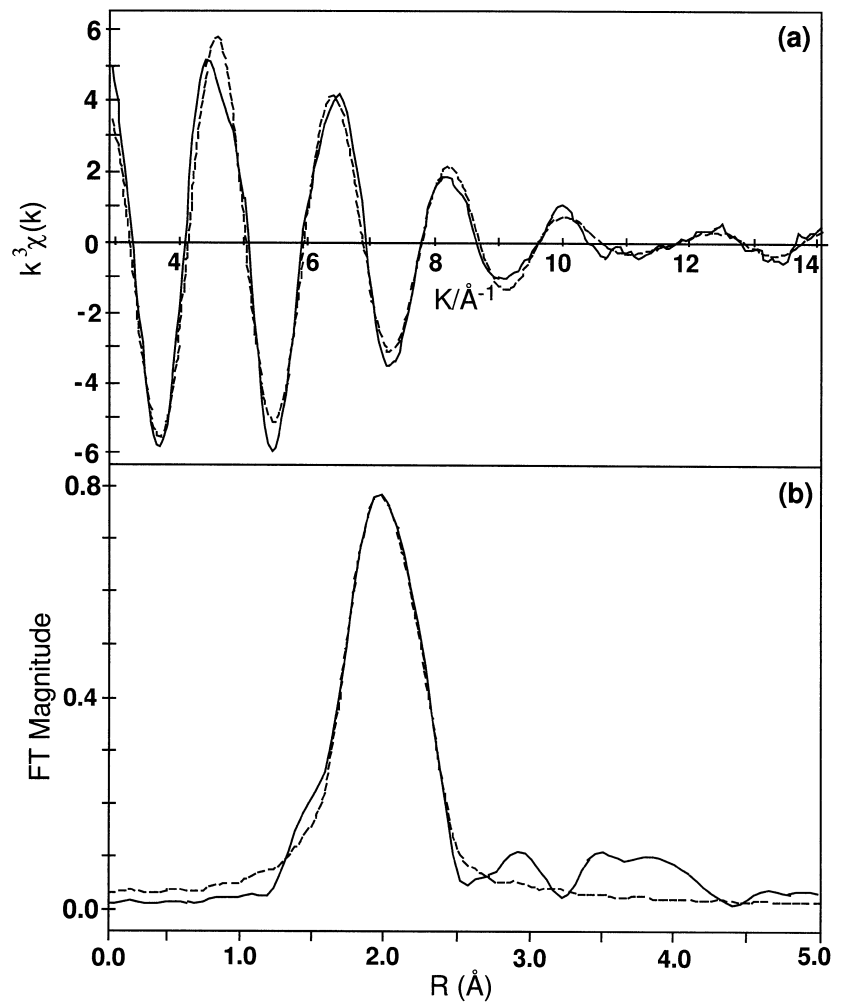

Fig. 3. (a) $k^{3}$-weighted experimental and least-squares fitted EXAFS of Zn-bacitracin A. (b) The corresponding Fourier transform (FT). The solid line shows the experimental data, and the broken line represents the calculated EXAFS and FT using the nearest $\mathrm{N}$ - and $\mathrm{O}$-neighbours $(3 \times \mathrm{Zn}-\mathrm{N}+\mathrm{Zn}-\mathrm{O})$. The final results are shown in Table 3.

(1.0-25.0 ̊). This filter removes low-frequency contributions to the EXAFS below $1 \AA$, but does not smooth the spectrum (i.e. the noise is not removed). The data were analysed over the range $k_{\min }=3$ to $k_{\text {max }}=14.0 \AA^{-1}$ and the refinement carried out to minimise the fit index $(\mathrm{FI})$.

$\mathrm{FI}=\sum_{i}\left\{k^{3}\left[\chi_{i}^{\prime}(k)-\chi_{i}(k)\right]\right\}^{2}$

Here $\chi_{i}^{\prime}(k)$ and $\chi_{i}(k)$ are the theoretical and experimental EXAFS respectively.

Fig. 3a shows the $k^{3}$-weighted zinc K-edge EXAFS data for bacitracin A and the theoretical curve produced from our model; Fig. 3b shows the Fourier transform. The parameters obtained from the leastsquares fitting are listed in Table 3 . The main contribution to the EXAFS arises from the atoms coordinated to zinc. This is also represented by the major peak centred at approx. $2.0 \AA$ in the magnitude of 
the Fourier transform; the width and asymmetry of this peak are consistent with a spread of distances from different backscatters.

Chemical knowledge enables us to define several options for the coordinate bonding to the ligand atoms: NSON, SONO, NSONO, NNON, NONO and NNONO (Table 1). The first three options involve sulphur as an immediate backscatter. The statistics on compounds containing $\mathrm{Zn}-\mathrm{S}$ contacts indicate that 2.3-2.5 $\AA$ may be a reasonable range for the $\mathrm{Zn}-\mathrm{S}$ distance in this system, as discussed above. Initial analysis of the EXAFS data showed that only light atoms are evident within a coordination sphere of radius $2.5 \AA$. Since we would expect the $\mathrm{Zn}-\mathrm{S}$ bond length in this system to be within this range, sulphur is initially rejected as a coordinating atom. This leaves us with models NNON, NONO and NNONO. The data were first fitted to a single peak (i.e. with a single distance and Debye-Waller factor), using the aforementioned $\mathrm{k}^{1}$ and $\mathrm{k}^{3}$ weighting schemes in order to yield the unique values for the overall coordination number and Debye-Waller factors. Assuming that $\mathrm{N}$ is the only backscatter in this single shell model a coordination number of 4.7 was obtained. This result is independent of the options in Table 1 derived from chemical knowledge. However, we know from other data discussed above that the oxygen from Glu-4 has to be found in the coordination sphere. Nitrogen under-represents the backscattering from oxygen, and substituting an $\mathrm{N}$ atom by an $\mathrm{O}$ atom reduces the estimated coordination number to 4.0. This result hence supports the NNON option in a tetra-coordinated complex. The results for the curve fitting are shown in Table 3.

The one-dimensional nature of EXAFS spectra means that splitting the single shell model into two shells requires careful consideration. This involves combining a statistical evaluation with chemical knowledge. The two-shell model, composed of oxygen and nitrogen atoms, yielded multiplicities 0.90 $(\mathrm{O})$ and $3.10(\mathrm{~N})$ for a coordination number of four (the latter was not restricted during the refinement). The result of the two-shell refinement was compared with the single shell model described above. The two-shell model is significant at the 99\% level [34] and the number of independent parameters was significantly less than the theoretical number of independent data points as given by the Nyquist equation, $N_{\text {ind }}=2 \Delta k \Delta r / \pi+1$, assuming that the EXAFS data are significant over $1-4 \AA$ [35]. In the final least-squares round chemical knowledge was also used to fix the multiplicities for nitrogen and oxygen to 3 and 1, respectively, to give the same total coordination number of four. This reduces both the number of refined parameters and their estimated standard deviations. The results of the refinement also include the $R$-factor, were $\chi_{i}^{\prime}(k)$ and $\chi_{i}(k)$ are the theoretical and experimental EXAFS respectively.

$R-$ factor $=\frac{\int\left|\left[\chi_{i}^{\prime}(k)-\chi_{i}(k)\right]\right| k^{3} \mathrm{~d} k}{\int\left|\chi_{i}(k)\right| k^{3} \mathrm{~d} k} \times 100 \%$

From the analysis we conclude that sulphur is not ligated to zinc and that the coordination consists of four light atoms as represented by the single shell model for NNON. Further, on adding a second shell refinement gives results that are consistent with the single oxygen and three nitrogens of this environment; the magnitudes of the Debye-Waller factors

Table 3

Results of EXAFS curve fitting for Zn-bacitracin A

\begin{tabular}{lllllll}
\hline Model & $R$-Factor $(\%)$ & FI & Interaction & $N$ & $R(\AA)$ & $2 \sigma^{2}\left(\AA^{2}\right)$ \\
\hline $\mathrm{Zn} \rightarrow \mathrm{N}, \mathrm{N}, \mathrm{O}, \mathrm{N}$ & 19.6 & 0.52 & $\mathrm{Zn}-\mathrm{N}$ & 3 & $2.096(3)^{\mathrm{a}}$ & 0.014 \\
$\mathrm{Zn} \rightarrow \mathrm{N}, \mathrm{S}, \mathrm{N}, \mathrm{N}$ & & & $\mathrm{Zn}-\mathrm{O}$ & 1 & $1.946(3)$ & 0.006 \\
& 24.4 & 1.20 & $\mathrm{Zn}-\mathrm{N}$ & 3 & $2.004(2)$ & 0.015 \\
\end{tabular}

Each distance $(R)$ is associated with a coordination number $N$ and thermal vibration (Debye-Waller-type factor) $2 \sigma^{2}$. The refined correction to the threshold energy of the absorption edge $E_{0}$ is $18.88 \mathrm{eV}$.

${ }^{a}$ The standard deviation in the least significant digit as calculated by EXCURV90 is given in parentheses. However, note that such estimates of precision overestimate the accuracy, particularly in cases of high correlation between parameters. The estimated standard deviations for distances $>2.5 \AA$ are $0.01 \AA$ with $\pm 20 \%$ accuracy for $2 \sigma^{2}$. 
$\left(2 \sigma^{2}\right)$ are also consistent with a single oxygen $(0.006$ $\AA^{2}$ ) and a spread of distances for the nitrogen atoms $\left(0.014 \AA^{2}\right)$.

In order to confirm this conclusion the data were also fitted to a NSNN model, which is comparable to the NNON model with respect to curve fitting and number of parameters. As nitrogen and oxygen are very similar in EXAFS, the NSNN model will also be representative of the NSON model of Table 1 . The results (see Table 3 ) show that the model involving coordination to $\mathrm{S}$ is inferior to the NNON model. The $R$-factor is higher, the fit index (FI) is higher, and the $\mathrm{Zn}-\mathrm{S}$ distance is too short compared both to the statistics in Table 2 and to the zinc sulphide model compound.

\section{Conclusions}

The experimental data from this EXAFS study correspond best to a model where the zinc ion in bacitracin $\mathrm{A}$ is coordinated by one oxygen and three nitrogen atoms in the first coordination shell. This model supports the hypothesis that the zinc ion is coordinated by the $\mathrm{N}$-terminal $-\mathrm{NH}_{2}$ group of Ile-1, the nitrogen atom of the thiazoline ring (from Cys2 ), the carboxylic side chain of Glu-4, and the imidazole side chain of His-10. It is also consistent with the statistical data from CSD (see Table 2), both with respect to distances, and with respect to the preference for $\mathrm{N}$-coordination in the thiazoline ring. It is not possible to completely rule out alternative solutions from these data, like whether the $\mathrm{N}$ terminal $-\mathrm{NH}_{2}$ group is really involved in coordination, rather than a group from a neighbouring bacitracin A molecule in the crystal. However, it seems unlikely that the sulphur atom of the thiazoline ring is involved in the coordination.

This result fits previous data on coordination of metal ions by bacitracin $\mathrm{A}$, with one important exception. The current data seem to indicate that the previous interpretation of the NMR data of Mosberg et al. [15] may be incorrect, and that the coordination of a zinc ion by the thiazoline ring introduces large shift effects in the protons close to sulphur, although the coordination is to nitrogen. It is possible that this is linked to the electronic resonance effects in thiazolines (see Fig. 2), and this is currently being investigated using $\mathrm{ab}$ initio calculations.

\section{Acknowledgements}

We thank Mr. K.-C. Cheung, station scientist at the Daresbury Laboratory, for providing beamtime. FD has been supported by a grant from the Norwegian Research Council (NFR 113733/420). Support from VISTA-Statoil to DGN is much appreciated.

\section{References}

[1] W.A. Toscano, D.R. Storm, in: D.J. Tipper (Ed.), Antibiotic Inhibitors of Bacterial Cell Wall Biosynthesis, Pergamon Press, Oxford, 1987, pp. 101-113.

[2] K.J. Stone, J.L. Strominger, Proc. Natl. Acad. Sci. USA 68 (1971) 3223-3227.

[3] D.R. Storm, Ann. NY Acad. Sci. 235 (1974) 387-398.

[4] R.E. Galardy, M.P. Printz, L.C. Craig, Biochemistry 10 (1971) 2429-2436.

[5] D. Konz, A. Klens, K. Schorgendorfer, M.A. Marahiel, Chem. Biol. 4 (1997) 927-937.

[6] R.H. Adler, J.E. Snoke, J. Bacteriol. 83 (1962) 1315-1317.

[7] D.R. Storm, J.L. Strominger, J. Biol. Chem. 248 (1973) 3940-3945.

[8] S. Pfeffer, W. Hohne, S. Branner, K. Wilson, C. Betzel, FEBS Lett. 285 (1991) 115-119.

[9] S. Pfeffer Hennig, Z. Dauter, M. Hennig, W. Hohne, K. Wilson, C. Betzel, Adv. Exp. Med. Biol. 379 (1996) 2941.

[10] N. Kobayashi, T. Takenouchi, S. Endo, E. Munekata, FEBS Lett. 305 (1992) 105-109.

[11] J.T. Garbutt, A.L. Morehouse, A.M. Hanson, Agric. Food Chem. 9 (1961) 285-289.

[12] L.C. Craig, W.F. Phillips, M. Burachik, Biochemistry 8 (1969) 2348-2356.

[13] N.W. Cornell, D.G. Guiney Jr., Biochem. Biophys. Res. Commun. 40 (1970) 530-536.

[14] R.E. Wasylishen, M.R. Graham, Can. J. Biochem. 53 (1975) $1250-1254$.

[15] H.I. Mosberg, D.A. Scogin, D.R. Storm, R.B. Gennis, Biochemistry 19 (1980) 3353-3357.

[16] D.A. Scogin, H.I. Mosberg, D.R. Storm, R.B. Gennis, Biochemistry 19 (1980) 3348-3352.

[17] E.G. Seebauer, E.P. Duliba, D.A. Scogin, R.B. Gennis, R.L. Belford, J. Am. Chem. Soc. 105 (1983) 4926-4929.

[18] D.A. Scogin, T.O. Baldwin, R.B. Gennis, Biochim. Biophys. Acta 742 (1983) 184-188.

[19] H.W. Park, S.R. Boduluri, J.F. Moomaw, P.J. Casey, L.S. Beese, Science 275 (1997) 1800-1804. 
[20] F.H. Allen, O. Kennard, Chem. Design Autom. News 8, (1) (1993) 31-37.

[21] K. Lonsdale, in: International Tables for X-Ray Crystallography, vol. 3, Kynick Press, Birmingham, 1962, p. 175.

[22] A. Bondi, J. Phys. Chem. 68 (1964) 441-451.

[23] E. Shuter, H.R. Hoveyda, V. Karunaratne, S.J. Rettig, C. Orvig, Inorg. Chem. 35 (1996) 368-372.

[24] P.A. Boo, M.D. Couce, E. Freijanes, J.S. Casas, A. Castiñeiras, A.S. González, J. Sordo, U. Russo, J. Organomet. Chem. 506 (1996) 253-258.

[25] M.M. Khalil, A.H.H. Elghandour, M. Mostafa, M.M. Shoukry, Polyhedron 13 (1994) 3295-3297.

[26] D.L. Eng-Wilmot, D. van der Helm, J. Am. Chem. Soc. 102 (1980) 7719-7725.

[27] M. Witanowski, W. Sicinska, Z. Biedrzycka, Z. Grabowski, G.A. Webb, J. Chem. Soc. Perkin Trans. 2 (1996) 619-623.
[28] N. Binsted, J.W. Campbell, S.J. Gurman, P.C. Stephenson, SERC Daresbury Laboratory, 1990.

[29] S.J. Gurman, N. Binsted, I. Ross, J. Phys. C Solid State Phys. 17 (1984) 143-151.

[30] N.N. Greenwood, A. Earnshaw, Chemistry of the Elements, Pergamon Press, Oxford, 1984, p. 1405.

[31] N. Burger, H. Fuess, Z. Kristall. 145 (1977) 346-355.

[32] W.R. Scheidt, J.U. Mondal, C.W. Eigenbrot, A. Adler, L.J. Radonovich, Inorg. Chem. 25 (1986) 795-799.

[33] F.W.H. Kampers, PhD thesis, Eindhoven University of Technology, 1988.

[34] W. Joyner, K.J. Martin, P. Meehan, J. Phys. C Solid State Phys. 20 (1987) 4005-4012.

[35] E.A. Stern, Phys. Rev. B 48 (1993) 9825-9827. 